\title{
Effect of Mould and Melt Temperature on The Properties of Styrene Acrylonitrile Moulded Parts
}

\author{
Cahyo Budiyantoro ${ }^{a}$, Adi Nugroho ${ }^{b}$ \\ ${ }^{a}$ Department of Mechanical Engineering, Universitas Muhammadiyah Yogyakarta \\ Jl. Lingkar Selatan, Yogyakarta 55183, Indonesia \\ Phone: (0274) 387656 /fax: 0274387646 \\ e-mail: cahyo_budi@umy.ac.id \\ ${ }^{b}$ Politeknik ATMI Surakarta \\ JI. Adisucipto/ Jl. Mojo No.1 Surakarta \\ Phone: (0271) 271714466 \\ e-mail: adi_nugroho@atmi.ac.id
}

\begin{abstract}
The quality of injection moulded parts can be influenced by processing parameters. The effect of mould temperature and melt temperature on the weight and shrinkage of Styrene Acrylonitrile moulded part was observed in this research. It was found that by increasing mold temperatures at constant melt temperature and injection speed, the weight of the part decreased and the shrinkage value slightly decreased. On the other hand, by increasing melt temperature at constant mold temperature and injection speed, the part weight increased. Both mold temperature and melt temperature gave only a small effect because the temperature difference was not too large.
\end{abstract}

Keywords: Mold temperature; melt temperature; SAN; injection molding; part weight; shrinkage

\section{INTRODUCTION}

The quality of injection molded parts can be affected by many factors including design paramater, material and processing condition. Shrinkage and part weight are some of important factors determining the quality of injection molded parts. Mustafa et.al [1] stated that determination of optimal melt temperature is necessary to obtain acceptable part quality and as the melt temperature increased the mold temperature increased accordingly. According to Postawa et al. [2], the changes in shrinkage and weight of injection molded parts is a function of processing conditions, not only packing pressure but also injection temperature has considerable effects on the shrinkage behavior of the parts produced. Mould temperature is controlled by circulation of cooling medium in the mould, and in fact the temperature of cooling medium is normally different with the mould surface temperature. Melt temperature is built by combination of barrel setting and material friction inside the barrel. The setting value of mold temperature and melt temperature are mainly determined by plastic material and part geometry. Shrinkage of injection molded products is most influenced by the holding pressure and the melt temperature, an increase in those parameters causes a decrease in shrinkage and the effects of injection velocity and mold temperature on shrinkage are much smaller and differ for each material (K. M. B. Jansen et. al [2]). The research of Alias Mohd et. al [3] verifing that the lowest shrinkage value was when applying lower injection pressure with maximum cooling time and at lower injection pressure with a lower melting temperature. The study of Nagahanumaiah [4] found that injection speed and melt temperature have significant influence on part weight and shrinkage 
Initially, to set the barrel temperature it is easier to use values that recommended by the resin manufacturer. The temperature of heating zones along the barrel can be adjusted to find suitable melting condition in the barrel and to get lower viscosity of the melt. Higher melt temperature will result in lower viscosity of the melt. When the viscosity is low, the melt will flow easily into the mould. Ideally, melt temperature entering and leaving the nozzle should be the same. The nozzle temperature setting will depend on the design of the nozzle, the type of resin and the shot size.

The task of this research is to determine the influence of mould temperature and melt temperature on the shrinkage and weight of the moulded parts.

\section{Methods}

\subsection{Equipment}

- Injection Moulding Machine : Meiki 70 tons

- Mould with 2 cavities, attached on the machine

- Cooling Device

- Data Acquisition Computer

- Edge cutter for separating the sprue

- Shrinkage Measurement Device: Mitutoyo Measuring Device

\subsection{Material}

The used material for this trial was Styrene Acrylonitrile (SAN): SAN Luran 368R from company BASF. Recommended processing parameters from material manufacturer are:

- Melt temperature $: 230-250^{\circ} \mathrm{C}$

- Mold temperature $: 40-80^{\circ} \mathrm{C}$.

\subsection{Test Methods}

Before running the trials, the ideal processing parameter should be obtained in order to set the process starting point. Table 2.1 shows the initial cylinder temperature, the highest temperature was set at $240{ }^{\circ} \mathrm{C}$ and then the heating band next to nozzle was set at $230^{\circ} \mathrm{C}$ following the recommendation of material manufacturer. This temperature should be reduced toward the hopper flange. In order to avoid early melting of plastic material, the hopper flange should be cooled at $60^{\circ} \mathrm{C}$.

Table 2.1. Initial Barrel Temperature

\begin{tabular}{cccccc}
\hline Zone & $\begin{array}{c}\text { Hopper } \\
\text { Flange }\end{array}$ & $\mathbf{1}$ & $\mathbf{2}$ & $\mathbf{3}$ & Nozzle \\
\hline Temperature $\left[{ }^{\circ} \mathbf{C}\right]$ & 60 & 210 & 220 & 230 & 240 \\
\hline
\end{tabular}

Table 2.2 shows the initial setting parameter of injection molding machine. The value of parameter was also taken from material data sheet. Injection pressure is the pressure applied on the injection screw when a material is being injected into the mold, high injection pressures may be required during mold filling part of the molding cycle in order to maintain the preferred mold filling speed. Once the cavity is filled, a holding pressure is maintained to compensate for material shrinkage. To achieve consistent plasticization of thermoplastic, the back pressure should be set properly and thereby enable accurate shot weight control. The melt cushion is the material at the front of the screw when the screw is in the forward position. Proper value of melt cushion will allow the part to pack out evenly. 


\begin{tabular}{lcc}
\hline & Table 2.2 Initial Setting Parameter & \\
\hline Paramater & Setting Value & Actual value \\
\hline Injection Pressure [bar] & 1150 & 1070 \\
Injection Speed [mm/s] & 30 & \\
Holding Pressure [bar] & 750 & 749 \\
Back Pressure [bar] & 70 & \\
Mould Temperature $\left[{ }^{\circ} \mathrm{C}\right]$ & 60 & 248 \\
Melt Temperature $\left[{ }^{\circ} \mathrm{C}\right]$ & 240 & 4.70 \\
Switch-over Point $[\mathrm{mm}]$ & 4.70 & 1.11 \\
Injection Time [s] & & 12.16 \\
Dosing Time [s] & & 26.8 \\
Cycle Time [s] & & 436 \\
Cavity Pressure [bar] & & 2.76 \\
Melt Cushion [mm] & & \\
\hline
\end{tabular}

By using initial setting parameters, the standard specimen were produced and they were used as reference specimens. Afterward, some parameters (injection speed, mould temperature, barrel temperature) were changed for every trial as follow:

Table 2.3. Setting parameter for trial

\begin{tabular}{cccccccc}
\hline $\begin{array}{c}\text { Trial } \\
\text { no. }\end{array}$ & $\begin{array}{c}\text { Injection } \\
\text { speed } \\
{[\mathbf{m m} / \mathbf{s}]}\end{array}$ & $\begin{array}{c}\text { Mould } \\
\text { temperature } \\
{\left[{ }^{\circ} \mathbf{C}\right]}\end{array}$ & $\begin{array}{c}\text { BH } \\
\text { MH3 }\end{array}$ & $\begin{array}{c}\text { MH2 } \\
{\left[{ }^{\circ} \mathbf{C}\right]}\end{array}$ & MH1 & EF \\
\hline 1 & 40 & 20 & 200 & 200 & 190 & 180 & 45 \\
2 & 20 & 20 & 200 & 200 & 190 & 180 & 45 \\
3 & 60 & 20 & 200 & 200 & 190 & 180 & 45 \\
4 & 40 & 40 & 200 & 200 & 190 & 180 & 45 \\
5 & 40 & 60 & 200 & 200 & 190 & 180 & 45 \\
6 & 40 & 20 & 220 & 220 & 210 & 200 & 45 \\
7 & 40 & 20 & 240 & 240 & 230 & 220 & 45 \\
\hline
\end{tabular}

For every trial, 15 cycles were carried out. The result from the first 10 cycles were ignored, they are considered as unstable condition for establishing the thermal equilibrium. The 5 last shots of every cycle were marked and then the weight, isochromatic lines and the shrinkage of the parts (processing shrinkage and after annealing) were analyzed. Before analyzing the results, annealing procedure was conducted on the specimens for 1 hour at $95^{\circ} \mathrm{C}$ in the oven.

\section{Results And Discussion}

\subsection{Influence of mould temperature on experimental values}

The cavity pressure increases as the mould temperature increased. In a hot mould surface, it is clear that by increasing the mold temperature the heat transfer from the melt to the mold walls will be delayed [5], plastic melt creates less frozen layers resulting low pressure drop in the cavity, as can be seen in Graphic 3.1. 


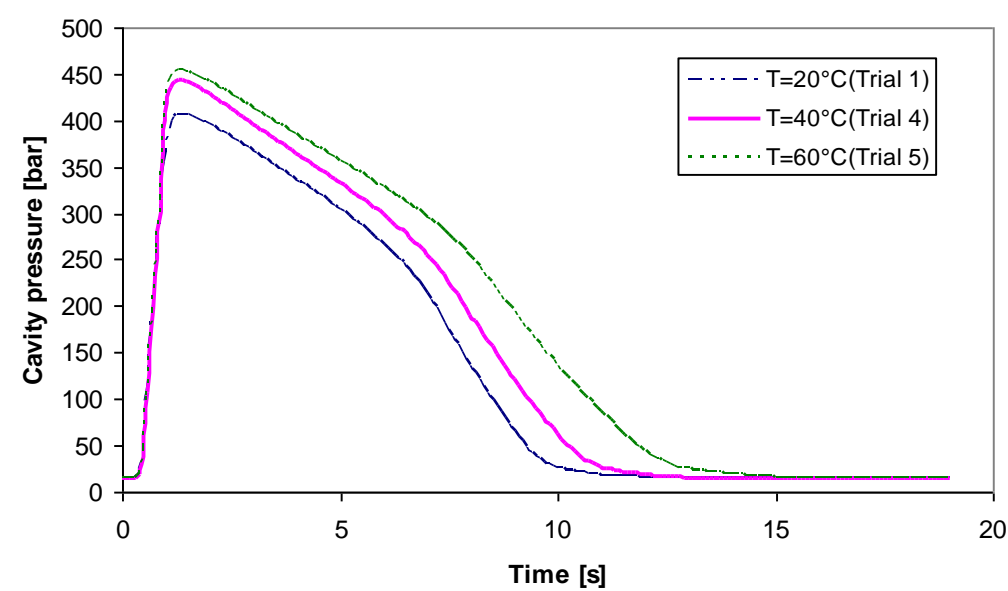

Graphic 3.1. Influence of mould temperature on cavity pressure profile

Graphic 3.2 shows that there is no significant differences in the hydraulic pressure profile when the mould temperature increased. Mould temperature does not have effect on the hydraulic pressure. The first peak occurs due to the resistance of the melt in the nozzle.

In graphic 3.3, constant maximum hydraulic pressure and cavity pressure were reached by increasing mould temperature.

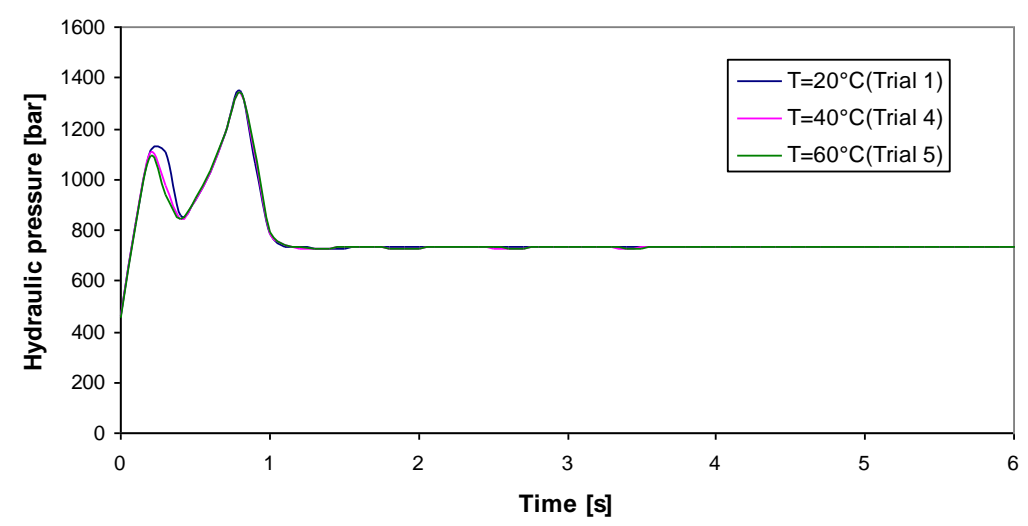

Graphic 3.2. Influence of mould temperature on hydraulic pressure profile

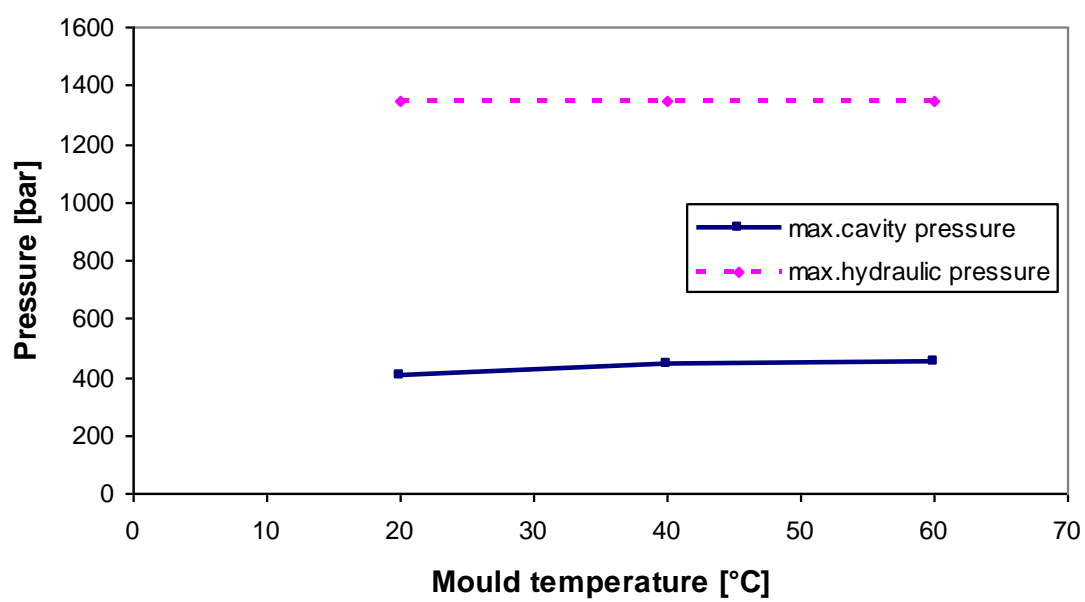

Graphic 3.3. Influence of mould temperature on max.hydraulic pressure and max.cavity pressure 
In graphic 3.4, it can be seen that part weight decreased when the mould temperature increased. However the differences between the maximum and the minimum are very small, it is only about $0,01 \mathrm{~g}$.

Increasing of mould temperature will decrease the longitudinal shrinkage. Higher mould temperature result in low orientation degree because the orientation have more time to relax. The shrinkage after annealing is higher than the shrinkage before annealing. After annealing, the residual orientation, which is frozen during cooling, can relax to the equilibrium state so materials shrink more after heating up. The differences of longitudinal shrinkage (before and after annealing) at higher mould temperature suppose to be less compared to the difference at lower mould temperature. See graphic 3.5 .

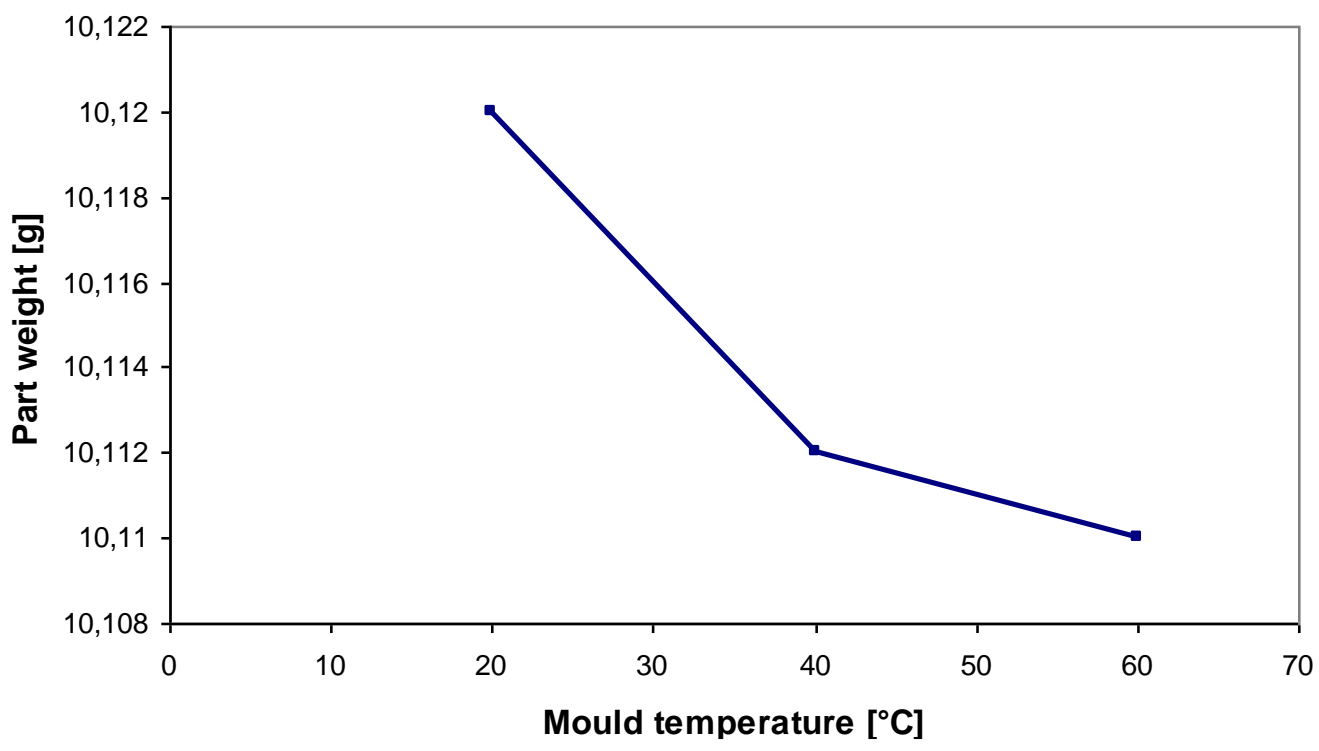

Graphic 3.4. Influence of mould temperature on part weight

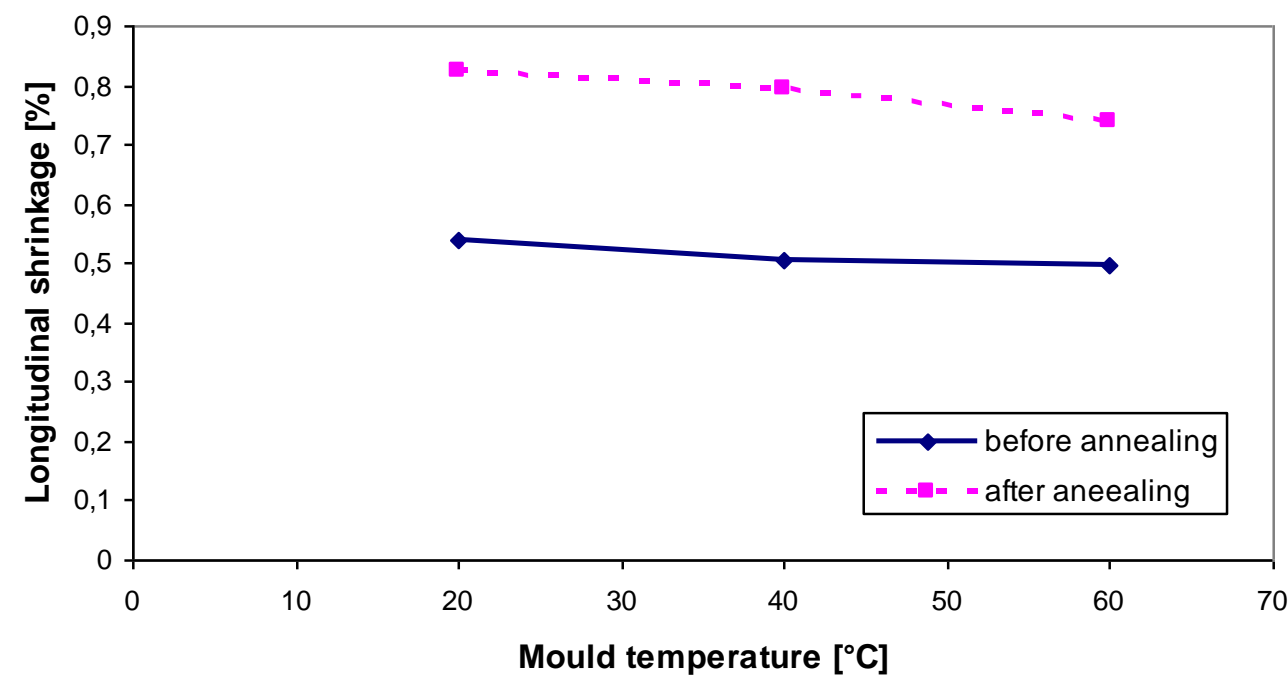

Graphic 3.5. Influence of mould temperature on longitudinal shrinkage

\subsection{Influence of melt temperature on experimental values}

Increase the barrel temperature will result in higher melt temperature. Increase the melt temperature will result in higher cavity pressure because it will lower down the viscosity of the melt and make easier flow to fill into the cavity. Due to the melt viscosity, it necessary to use higher hydraulic pressure when the melt temperature is low. Normally in hydraulic

JEMMME | Journal of Energy, Mechanical, Material, and Manufacturing Engineering 
pressure profile, only one peak should be visible. However the results obtained from the trials show a double peak. The first peak occurs due to the resistance in the nozzle. See Figure 13, 14 and 15.

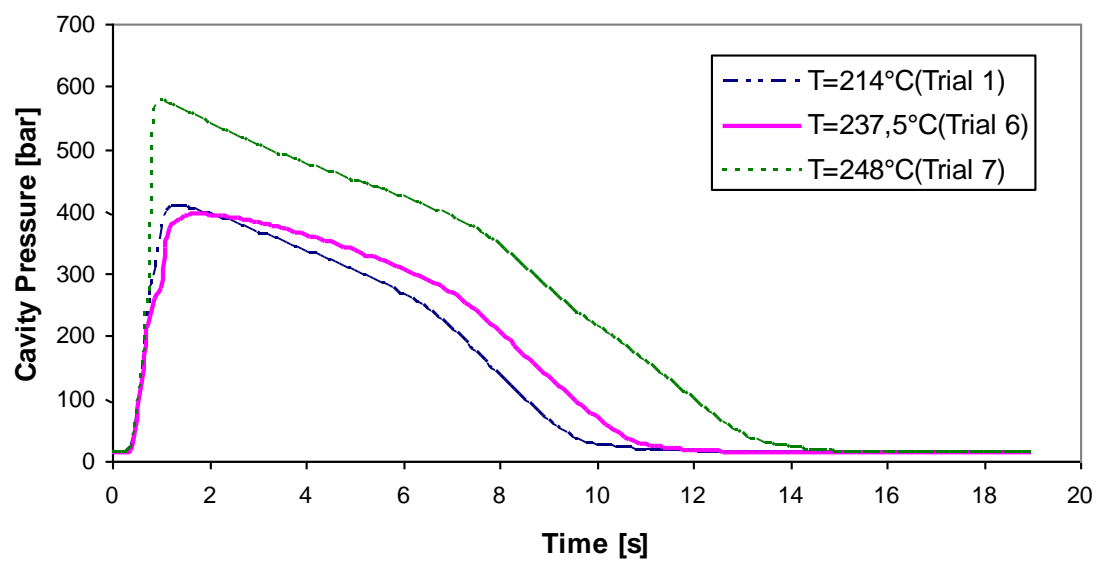

Graphic 3.6 Influence of melt temperature on cavity pressure profile

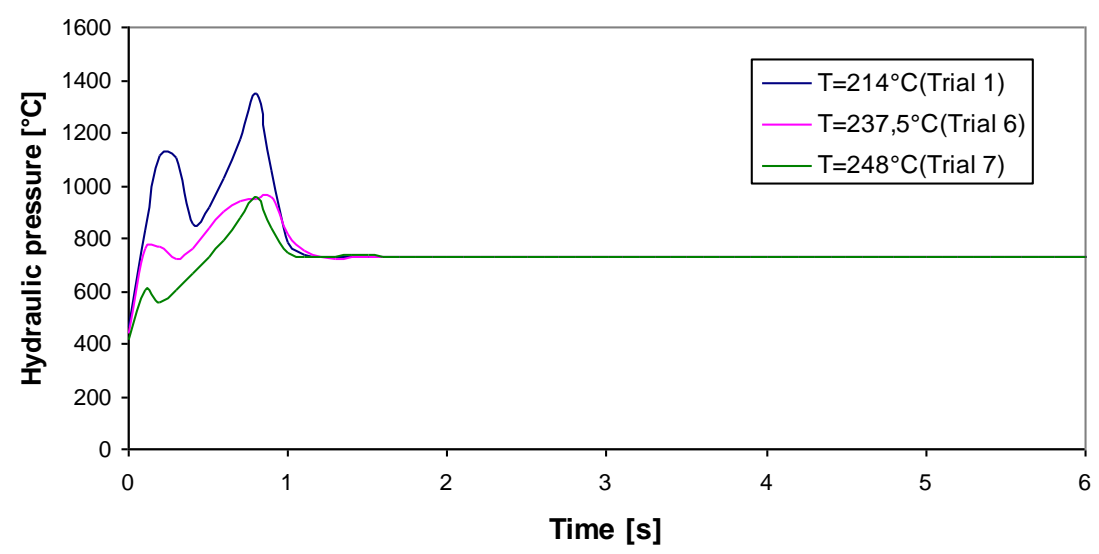

Graphic 3.7 Influence of melt temperature on hydraulic pressure profile

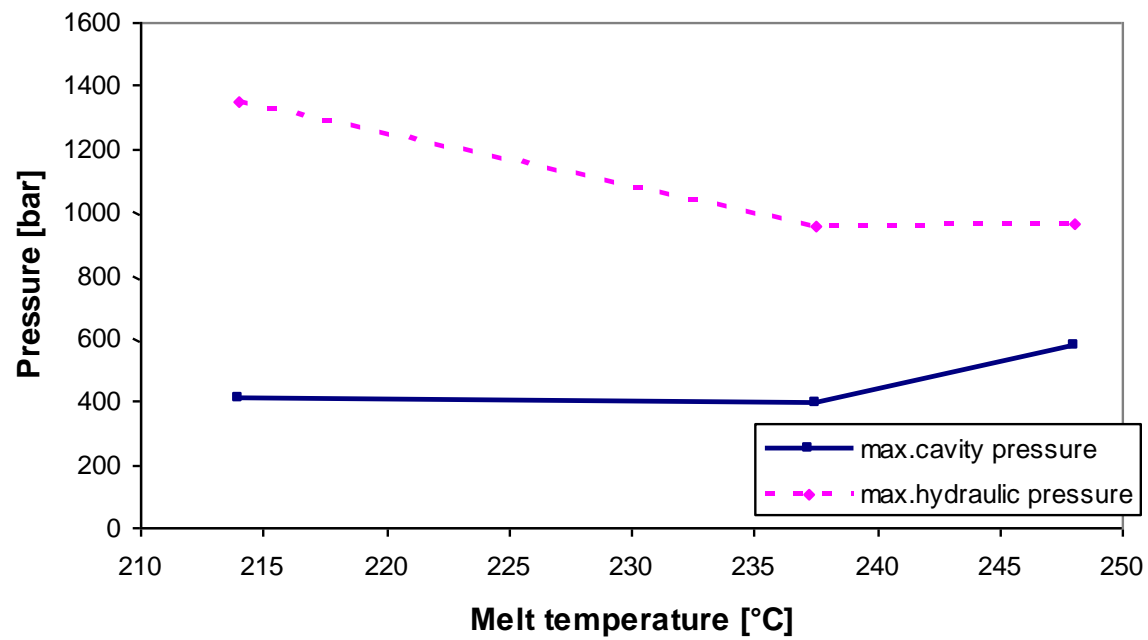

Graphic 3.8. Influence of melt temperature on maximum hydraulic pressure and cavity pressure 
It can be clearly seen that part weight will slightly increase when the melt temperature increases because more material can be flow into cavity easily since the viscosity of the melt is lower (see Figure 16). Higher melt temperature will result in lower density of the material.

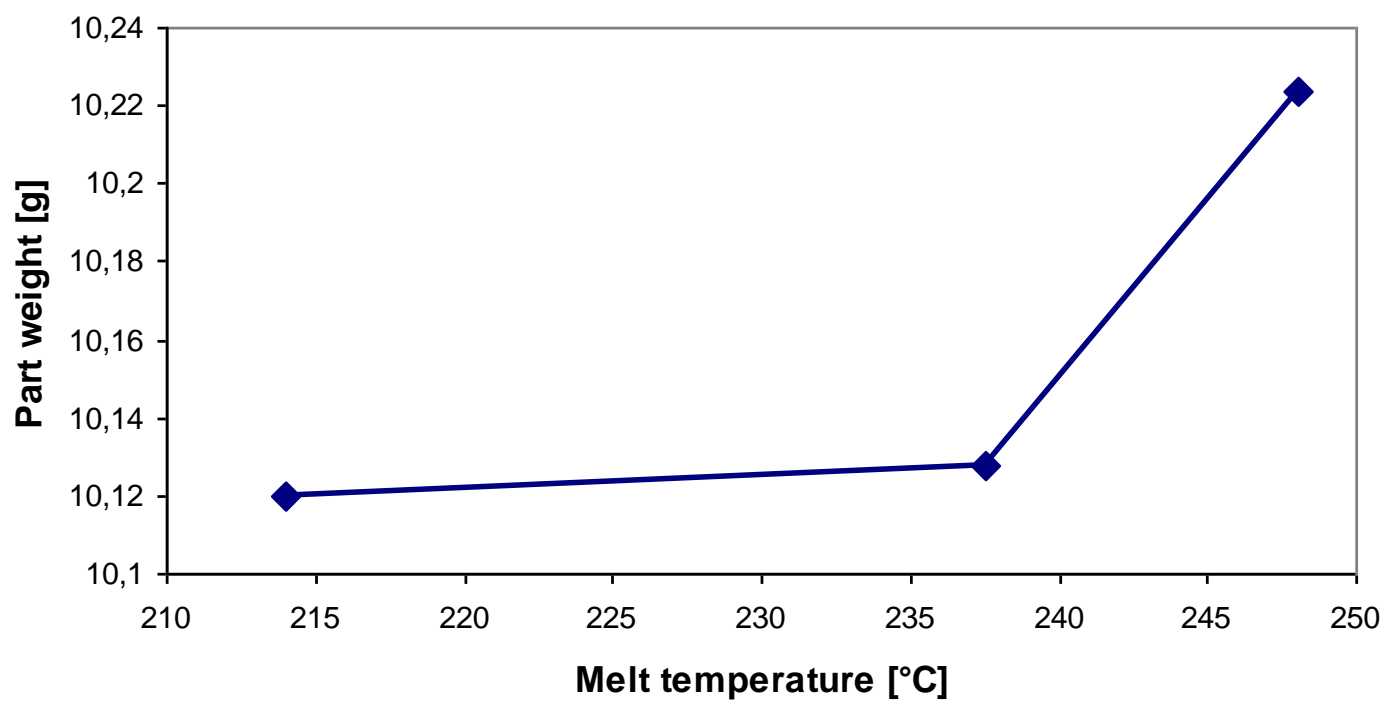

Graphic 3.9. Influence of melt temperature on part weight

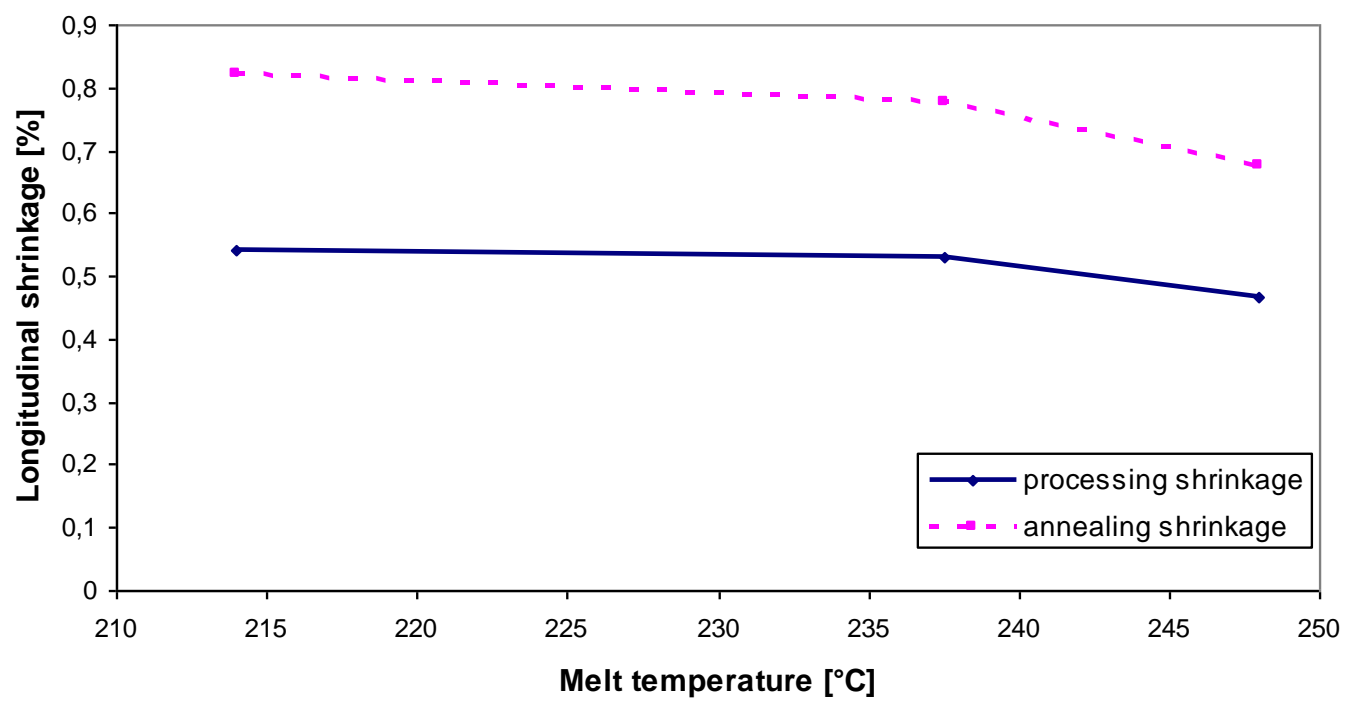

Graphic 3.10. Influence of melt temperature on longitudinal shrinkage

Increasing of melt temperature will decrease the longitudinal shrinkage due to less orientation degree, since the orientations have more time to relax and hence the remaining orientation in the part will be less. The lowest possible plastic melt-temperature that permits good molded parts will tend to produce less shrinkage [6]. The longitudinal shrinkage after annealing is more than the shrinkage before annealing, due to heat up which make the residual orientation, which is frozen during cooling, can relax to the equilibrium state. The difference in longitudinal shrinkage (before and after annealing) at higher melt temperature is less compared to the one at low melt temperature. 


\section{CONCLUSION}

Increasing mould temperature and melt temperature will give a significant effect on some parameters for example cavity pressure, hydraulic pressure, part weight, and also shrinkage as described in the analysis above.

Longitudinal shrinkage (shrinkage in flow direction) will decrease when the mould temperature is increased. The longitudinal shrinkage can also be decrease by increasing the injection speed and the melt temperature. Annealing (heat up) will result in higher longitudinal shrinkage of the part, because the residual orientation, which is frozen during cooling, can relax to the equilibrium state so materials shrink more after heating up. The difference of longitudinal shrinkage (before and after annealing) is more at lower injection speed, lower mould temperature and lower melt temperature.

\section{REFERENCES}

[1] Kurt, Mustafa., Kaynak, Yusuf., Kamber, Omer., Influence of molding conditions on the shrinkage and roundness of injection molded parts. The International Journal of Advanced Manufacturing Technology. 2010;46: 571 - 578.

[2] K. M. B. Jansen., D. J. Van Dijk, and M. H. Husselman., Effect of Processing Conditions on Shrinkage in Injection Molding. Polymer Engineering And Science. 1998, Vol. 38, No. 5

[3] Alias Mohd., Ainul Ayunie Roslan., Nor Bahiyah Baba., Effect of Injection Molding Parameters on Recycled ABS ( $r-A B S$ ) Mechanical Properties. Indian Journal of Science and Technology, Vol 9(9)

[4] Nagahanumaiah., B. Ravi., Effects of injection molding parameters on shrinkage and weight of plastic part produced by DMLS mold. Rapid Prototyping Journal. 2009., Vol 15(3):179-186

[5] Parvin Shokri., Naresh Bhatnagar., Effect of Packing Pressure and Mold Temperature on Fiber Orientation in Injection Molding Of Reinforced Plastics. The 8th International Conference on Flow Processes in Composite Materials (FPCM8). Douai, FRANCE. 2006; 409 - 416.

[6] J. M. Fischer, Handbook of Molded Part Shrinkage and Warpage, Plastic Design Library / William Andrew / Inc., USA, 2002 